Discrete Comput Geom 38:273-288 (2007)

DOI: $10.1007 / \mathrm{s} 00454-007-1338-3$

\title{
Covering a Triangle with Positive and Negative Homothetic Copies*
}

\author{
Zoltán Füredi \\ Department of Mathematics, University of Illinois at Urbana-Champaign, \\ Urbana, IL 61801, USA \\ z-furedi@math.uiuc.edu \\ and \\ Rényi Institute of Mathematics, Hungarian Academy of Sciences, \\ P.O. Box 127, Budapest, H-1364 Hungary \\ furedi@ renyi.hu
}

\begin{abstract}
Let $\Delta_{0}$ be a triangle and let $\mathcal{H}=\left\{\Delta_{1}, \ldots, \Delta_{n}\right\}$ be a set of homothetic copies of $\Delta_{0}, \Delta_{i}=x_{i} \Delta_{i}, x_{1} \geq x_{2} \geq \cdots \geq x_{n} \geq 0$. We prove that $\sum x_{i}^{2} \geq 1+x_{2}$ implies that there are positive and negative signs $\varepsilon_{1}, \ldots, \varepsilon_{n}= \pm 1$ and there exist translates of $\varepsilon_{1} \Delta_{1}, \ldots, \varepsilon_{n} \Delta_{n}$ that cover $\Delta_{0}$.

This result is used to answer a problem of $\mathrm{Xu}$ et al. [16]. We show that if $\Delta_{0}$ is the isosceles right triangle and if the total area of $\mathcal{H}$ is at least $(1+\sqrt{2}) / 2=1.207 \ldots$ times the area of $\Delta_{0}$, then there exist rotations $\rho_{i}$, each of them multiples of $45^{\circ}$, and translates of $\rho_{1} \Delta_{1}, \ldots, \rho_{n} \Delta_{n}$ that cover $\Delta_{0}$.
\end{abstract}

\section{Translation Coverings}

Let $C$ be a disk, i.e., convex, compact set on the Euclidean plane with interior points. Let $\mathcal{H}=\left\{C_{1}, \ldots, C_{i}, \ldots\right\}$ be a finite sequence of disks. We say that $\mathcal{H}$ permits a translation covering of $C$ if there exist translations $\tau_{i}$ such that $C \subseteq \bigcup_{i} \tau_{i}\left(C_{i}\right)$. Moser and Moon [12] showed that if $Q$ is the unit square and $\mathcal{H}$ is a set of squares of sizes $x_{1}, x_{2}, \ldots$ with total area $\sum_{i} x_{i}^{2} \geq 3$ and with sides parallel (or orthogonal) to $Q$, then $\mathcal{H}$ permits a translation covering of $Q$. This is the best possible bound as one can see from the example $x_{1}=x_{2}=x_{3}=1-\varepsilon, x_{4}=\cdots=0$.

L. Fejes Tóth proposed the following more general question. Suppose that each $C_{i}$ is a (positive) homothetic copy of $C$. How large must the sum of areas of the $C_{i}$ 's be,

* This research was supported in part by the Hungarian National Science Foundation under Grants OTKA 062321 and 060427 and by the National Science Foundation under Grant NFS DMS 06-00303. 
so that $C$ can be covered by translates of the $C_{i}$ 's? Denote the ratio of this minimum (infimum) and the area of $C$ by $f(C)$. The above cited theorem of Moon and Moser states $f(Q)=3$. This easily implies $f(C) \leq 12$ for every disk [3] and it was recently improved to $f(C) \leq 6.5$ by Januszewski [10]. One can observe that for any $C$ one has $f(C) \geq 2$ (two copies of size $1-\varepsilon$ cannot cover a diameter of $C$ ). Bezdek and Bezdek [3] conjectured that this is achievable for any triangle $\Delta$. This has been recently established by the present author [7] as

$$
f(\Delta)=2
$$

Small Sets. In fact, in the case of the unit square, $Q$, Moon and Moser showed that for $x_{1} \geq \cdots \geq x_{n}$ the total area

$$
\sum x_{i}^{2} \geq 1+2 x_{1}
$$

ensures a translation covering. Analyzing their proof one can see that $1+x_{1}+x_{2}$ is sufficient. This was generalized in [7] as follows.

Denote a convex body by $C \subset \mathbf{R}^{2}$, the infimum of the densities of coverings $\mathbf{R}^{2}$ by translates of $C$ by $\vartheta_{T}(C)$, and the family of all copies of $C$ whose positive homothety ratios are at most $\delta$ by $\mathcal{F}_{\delta}(C)$. For every $\varepsilon>0$ there exists a positive $\delta=\delta(\varepsilon, C)$ such that if the total area of sets from any family $\mathcal{H}(C) \subset \mathcal{F}_{\delta}(C)$ is at least

$$
\left(\vartheta_{T}(C)+\varepsilon\right) \operatorname{Area}(C),
$$

then there are translates of the sets from $\mathcal{H}(C)$ which cover $C$.

Large Sets. It is known that $\vartheta_{T}(C) \leq \frac{3}{2}$ for every planar disk (this upper bound is due to Besicovitch [2] although usually attributed to Fáry [5]) and this density can be obtained by a hexagonal lattice arrangement. See, e.g., the excellent monograph by Pach and Agarwal [13]. It is also conjectured that $\vartheta_{T}(\Delta)=\frac{3}{2}$ (see, e.g., [6]). Obviously, $\vartheta_{T}(Q)=1$. The theorem mentioned in (3) says that if the copies of $C$ in $\mathcal{H}$ are small, then good coverings can be constructed, so, ironically, $f(\Delta)$ and $f(Q)$ are large (2 and 3 , resp.) because $\mathcal{H}$ can contain large copies. Apparently, large copies mean large waste. This happens also in the coverings discussed below.

\section{Translations and Rotations}

Suppose that $\mathcal{H}$ consists of positive and negative homothetic copies of a triangle $\Delta_{0}$, then a total area at least $4 \operatorname{Area}\left(\Delta_{0}\right)$ ensures a translation covering, and here the constant 4 is the best possible. This was conjectured by Böröczky and proved by Januszewski [9]. The more special case of $\mathcal{H}$ being a finite sequence purely of homothetic copies of $-\Delta_{0}$ was proved earlier by Vásárhelyi [14]. She also considered translation coverings of the triangle $\Delta_{0}$ when $\mathcal{H}$ consists of homothetic copies, each of them rotated by a certain angle $\varphi$, see [15]. Here we consider a more general problem.

Definition 1. Let $C$ be a disk, let $\mathcal{H}=\left\{C_{1}, \ldots, C_{i}, \ldots\right\}$ be a finite sequence of disks, and let $\Gamma$ be a set of planar motions (usually a group of rotations). Let $\tau_{i}$ be a translation 
and let $\gamma_{i} \in \Gamma$, then the system $\left\{\tau_{1}\left(\gamma_{1} C_{1}\right), \tau_{2}\left(\gamma_{2} C_{2}\right), \ldots\right\}$ is called a $\Gamma$-translation of $\mathcal{H}$. We say that $\mathcal{H}$ permits a $\Gamma$-covering of $C$ if there exist translations $\tau_{i}$ and motions $\gamma_{i} \in \Gamma$ such that $C \subseteq \bigcup_{i} \tau_{i}\left(\gamma_{i} C_{i}\right)$.

Suppose that each $C_{i}$ is a (positive) homothetic copy of $C$. How large must the sum of areas of the $C_{i}$ 's be, so that $C$ can be covered by $\Gamma$-translates of the $C_{i}$ 's? Denote the ratio of this minimum (infimum) and the area of $C$ by $f_{\Gamma}(C)$. Denote the infimum of the densities of coverings of the plane by $\Gamma$-translated congruent copies of $C$ by $\vartheta_{\Gamma}(C)$.

Theorem 1. For every $\varepsilon>0$ there exists a $\delta=\delta(\varepsilon, C, \Gamma)>0$ such that the following holds. If $\mathcal{H}$ is a set of (positive) homothetic copies of $C$ and each member of $\mathcal{H}$ is smaller than $\delta C$ and for the total area we have

$$
\sum_{H \in \mathcal{H}} \operatorname{Area}(H) \geq\left(\vartheta_{\Gamma}(C)+\varepsilon\right) \operatorname{Area}(C),
$$

then there exist $\Gamma$-translates of the members of $\mathcal{H}$ that cover $C$.

The proof is a bit technical but straightforward, one can follow the argument in [7], we omit it. Naturally, a similar statement holds for every (finite) dimension.

$\varepsilon$ - and $\rho$-Coverings. The cases $\Gamma=$ rotations of integer multiples of $\pi$ \} and $\Gamma=$ \{rotations of integer multiples of $\pi / 4$ \} are called $\varepsilon$ - and $\rho$-coverings, respectively.

Theorem 2. Let $\Delta_{0}$ be a triangle and let $\mathcal{H}=\left\{\Delta_{1}, \ldots, \Delta_{n}\right\}$ be a set of homothetic copies of $\Delta_{0}, \Delta_{i}=x_{i} \Delta_{i}, x_{1} \geq x_{2} \geq \cdots \geq x_{n}>0$. Suppose that

$$
\sum x_{i}^{2} \geq 1+x_{2}
$$

Then there are positive and negative signs $\varepsilon_{1}, \ldots, \varepsilon_{n} \in\{1,-1\}$ and there exist translates of $\varepsilon_{1} \Delta_{1}, \ldots, \varepsilon_{n} \Delta_{n}$ that cover $\Delta_{0}$.

Unlike in the Moon-Moser theorem (2) here the second-order term depends on $x_{2}$. This theorem implies $f_{\varepsilon}\left(\Delta_{0}\right)=2$, but we have already known this from (1), since $f_{\varepsilon} \leq f$. Using Theorem 2 we answer a problem of Xu et al. [16]. They investigated $f_{\rho}\left(R_{0}\right)$ where $R_{0}$ is a right isosceles triangle. Let $\mathcal{H}=\left\{R_{1}, \ldots, R_{n}\right\}, R_{i}=x_{i} R_{0}$ and $x_{1} \geq x_{2} \geq \cdots \geq x_{n}>0$. They showed that if

$$
\sum_{i} x_{i}^{2} \geq \frac{8+\sqrt{8}}{7}+\sqrt{2} x_{1} \sim 1.56+1.41 x_{1},
$$

then $\mathcal{H}$ permits a $\rho$-cover. Theorem 2 clearly supersedes this. Here we also determine $f_{\rho}\left(R_{0}\right)$, it is $c:=(1+\sqrt{2}) / 2=1.2071 \ldots$

Theorem 3. Let $R_{0}$ be the triangle with vertices $(0,0),(a, 0)$ and $(0, a)$ and let $\mathcal{H}=$ $\left\{R_{1}, \ldots, R_{n}\right\}$ be a set of homothetic copies of $R_{0}$. Suppose that

$$
\sum_{i \geq 1} \operatorname{Area}\left(R_{i}\right) \geq \frac{1+\sqrt{2}}{2} \operatorname{Area}\left(R_{0}\right) .
$$


Then there are triangles $R_{i}^{\prime}$ congruent to $R_{i}$ and with sides parallel or orthogonal to some sides of $R_{0}$ such that $R_{0} \subseteq \bigcup_{i \geq 1} R_{i}^{\prime}$.

On the other hand, to establish the lower bound for $f_{\rho}(R)$ one needs, for every $\eta>0$, a sequence $x_{1}, \ldots, x_{n}$ with $\sum x_{i}^{2}>(c-\eta) a^{2}$ such that $\left\{x_{1} R_{1}, \ldots, x_{n} R_{n}\right\}$ cannot $\rho$-cover $R_{0}$. It is not difficult to see that one can take

$$
x_{k+1}:=(1-\eta / 2)(\sqrt{2}-1)^{k}, \quad \text { where } k=0,1,2, \ldots
$$

In Sections 3-5 we show how to $\varepsilon$-cover $\Delta_{0}$, i.e., Theorem 2 . In Sections $6-8$ we prove Theorem 3 showing $f_{\rho}\left(R_{0}\right) \leq c$. The uninteresting proof of $f_{\rho}\left(R_{0}\right) \geq c$ is omitted.

\section{Covering a Strip of $\Delta_{0}$}

The aim of this and the following two sections is to prove Theorem 2. We will define a translation and a rotation (of $0^{\circ}$ or $180^{\circ}$ ) of each member of $\mathcal{H}$. The procedure also supplies an algorithm with linear running time after ordering the triangles by their sizes. As $\varepsilon$-translation coverings are affine invariant, we may suppose that $\Delta_{0}$ (and all members of $\mathcal{H})$ are isosceles, right triangles. Suppose that the vertices of $\Delta_{0}$ are $(0,0),(a, 0)$, and $(0, a)$.

First, we prove the following lemma (in the second term there is an $x_{1}$ not $x_{2}$ as in Theorem 2). Let $\mathcal{H}=\left\{\Delta_{1}, \ldots, \Delta_{n}\right\}$ be a set of homothetic copies of $\Delta_{0}$ with side lengths

$$
a \geq x_{1} \geq x_{2} \geq \cdots \geq x_{n} \geq 0
$$

Suppose that

$$
x_{1}+x_{2}+\cdots+x_{n} \geq 2 a \text {. }
$$

Lemma 4. There exists a trapezoid $T$ of height $h$ on the bottom of $\Delta_{0}$, its vertices are $(0,0),(a, 0),(0, h)$, and $(a-h, h)$, and there exists an $s \geq 1$ such that the following two properties hold:

- An $\varepsilon$-translation of the largest $2 s+1$ triangles $\left\{\Delta_{1}, \ldots, \Delta_{2 s+1}\right\}$ covers $T$.

- In case of $h<$ a the covering of $T$ is economical, that is

$$
2 \operatorname{Area}(T) \geq \sum_{1 \leq i \leq 2 s+1} 2 \operatorname{Area}\left(\Delta_{i}\right)-a x_{1}+(a-h) x_{2 s+1} .
$$

Proof. If $x_{1}=a$ or $x_{1}=0$, then there is nothing to prove so we may suppose

$$
a>x_{1}>0 .
$$

Define $s$ as the smallest integer with

$$
a \leq x_{1}+\sum_{1 \leq i \leq s}\left(x_{2 i}+x_{2 i+1}-x_{2 s+1}\right) .
$$




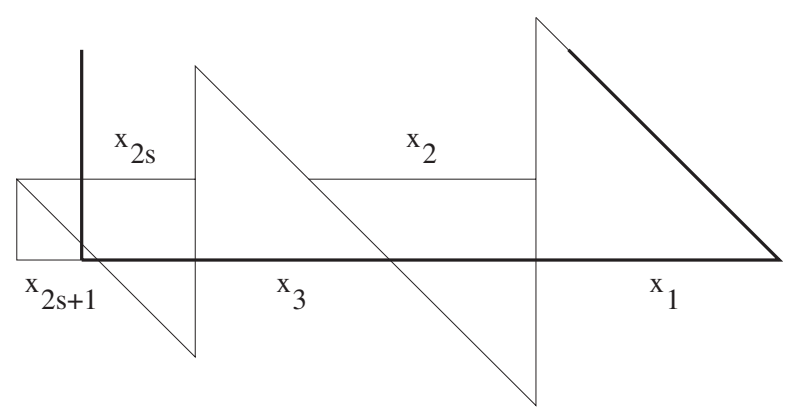

Fig. 1. $\Delta_{1},-\Delta_{2}, \ldots,-\Delta_{2 s}, \Delta_{2 s+1}$ cover a trapezoid of height $x_{2 s+1}$.

We claim that such an $s$ exists and $1<2 s+1 \leq n$. Obviously, $s$ must be at least 1 . On the other hand, the opposite of (9) and monotonicity (6) imply that

$$
a-x_{1}>x_{2}+x_{4}+\cdots+x_{2 s} \geq x_{3}+x_{5}+\cdots+x_{2 s+1},
$$

so $2 a-x_{1}>\sum_{i \leq 2 s+1} x_{i}$. Thus $2 a>\sum_{i \leq 2 s+2} x_{i}$ and (7) implies $2 s+3 \leq n$. So two more $x_{i}$ 's can be joined to $\left\{x_{1}, \ldots, x_{2 s+1}\right\}$ and in finitely many steps we reach an $s$ satisfying (9).

Let $\mathcal{R}$ denote the set of the largest $2 s+1$ triangles from $\mathcal{H}$. Geometrically, (9) means that one can translate $\Delta_{1},-\Delta_{2}, \ldots,(-1)^{i+1} \Delta_{i}, \ldots, \Delta_{2 s+1}$ such that they cover a trapezoid of height $x_{2 s+1}$ and base exceeding $a$, see Fig. 1 .

By definition

$$
a \geq x_{1}+\sum_{1 \leq i \leq s-1}\left(x_{2 i}+x_{2 i+1}-x_{2 s-1}\right) .
$$

We will use the following consequence of (6) and (10):

$$
x_{1}+x_{2}+\cdots+x_{s} \leq a .
$$

Now we are ready to define the trapezoid $T$ and its $\varepsilon$-translation covering by $\mathcal{R}$. The only thing needed to define $T$ is its height $h$. It is obtained from the following equation:

$$
x_{1}+\sum_{1 \leq i \leq s}\left(x_{2 i}+x_{2 i+1}-h\right)=a .
$$

Comparing (9) and (12) we obtain that

$$
x_{2 s+1} \leq h .
$$

Inequalities (6) and (11) imply $\sum_{1 \leq i \leq 2 s+1} x_{i} \leq(2 s+1) a / s$. This and (12) give

$$
h \leq \frac{s+1}{s^{2}} a .
$$

We claim that $\mathcal{R}$ can $\varepsilon$-cover $T$. Place the apex vertex of $\Delta_{1}$ (the vertex with the right angle) to the point $\left(a-x_{1}, 0\right)$, its other two vertices are $\left(a-x_{1}, x_{1}\right)$ and $(a, 0)$. For 


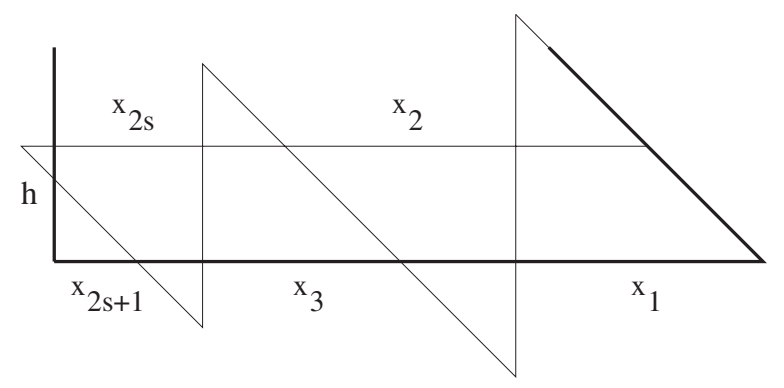

Fig. 2. The trapezoid $T$ of height $h$ is covered by $\Delta_{1}, \ldots, \Delta_{2 s+1}$.

$1 \leq i \leq s$ the triangles $-\Delta_{2 i}$ and $\Delta_{2 i+1}$ can form a rectangle of height $h$ and base length $\left(x_{2 i}+x_{2 i+1}-h\right)$. In the case $i=s$ this base might be negative, but as the sum of the base lengths is $a-x_{1}$ by (12) the $s$ rectangles can be put next to each other to cover a rectangle of size $\left(a-x_{1}\right) \times h$, see Fig. 2 .

Informally Fig. 2 is obtained from Fig. 1 by pushing the triangles horizontally closer to each other so they can cover a strip wider than $x_{2 s+1}$. More precisely, for $0 \leq i \leq s$ place $\Delta_{2 i+1}$ such that its apex vertex lies on the line $y=0$, it is at the point

$$
\left(\sum_{i<j \leq s}\left(x_{2 j}+x_{2 j+1}-h\right), 0\right),
$$

and the triangles $-\Delta_{2 i}$ are put below the line $y=h$, their apex vertices are at

$$
\left(\sum_{i \leq j \leq s}\left(x_{2 j}+x_{2 j+1}-h\right), h\right) \text {. }
$$

We obtained that $\bigcup_{\Delta_{i} \in \mathcal{R}} \varepsilon_{i} \Delta \supseteq T$, where $\varepsilon_{i}=(-1)^{i+1}$.

In the case of $h \geq a$ (by (14) this could happen only if $s=1$ ), $\mathcal{R} \varepsilon$-covers the whole $\Delta_{0}$, so our procedure stops. To finish the proof of the lemma in case of

$$
h \leq a
$$

we have to verify inequality (8). The proof of this inequality is purely algebraic, it does not use any geometry, therefore it is postponed to the next section as Lemma 5.

\section{Proof of an Inequality}

The following lemma implies (8).

Lemma 5. Suppose that $a \geq x_{1} \geq x_{2} \geq \cdots \geq x_{n} \geq 0$ and $a \geq h \geq x_{2 s+1}$ are real numbers and $s \geq 1$ is an integer satisfying (10) and (12), i.e.,

$$
\begin{array}{r}
x_{1}+\sum_{1 \leq i \leq s-1}\left(x_{2 i}+x_{2 i+1}-x_{2 s-1}\right) \leq a, \\
x_{1}+\sum_{1 \leq i \leq s}\left(x_{2 i}+x_{2 i+1}-h\right)=a .
\end{array}
$$


Then

$$
-\left(\sum_{i=1}^{2 s+1} x_{i}^{2}\right)+a x_{1}+2 a h-h^{2}-a x_{2 s+1}+h x_{2 s+1} \geq 0 .
$$

Proof. It is high school algebra, but because the domain of $F$ has many faces we have to distinguish many cases. First, as we have seen above, elementary calculation shows that the inequalities (6), (10), (12), (13) and (15) (appearing in the above lemma) imply that (9), (11), and (14) must hold, too.

Apply (6), (15), and (13), and then (12). We obtain

$$
\begin{aligned}
F\left(a, x_{1}, \ldots, x_{2 s+1}, h\right) & :=-\left(\sum x_{i}^{2}\right)+a x_{1}+a h+(a-h)\left(h-x_{2 s+1}\right) \\
& \geq-x_{1}\left(\sum x_{i}\right)+a x_{1}+a h \\
& =-x_{1}(a+s h)+a x_{1}+a h=h\left(a-s x_{1}\right) .
\end{aligned}
$$

This implies that $F \geq 0$ for $s x_{1} \leq a$, which is always true if $s=1$.

Suppose that $F$ can take negative values. Since it is homogeneous of degree 2, it takes a negative value with all variables at most 1 . Since $F$ is continuous on a compact part of the $(2 s+3)$-dimensional hypercube $[0,1]^{2 s+3}$, it takes its minimum, say at the point $\left(a, x_{1}, \ldots, x_{2 s+1}, h\right)$. We claim that, for these values,

$$
a>x_{1}>0, \quad a>h>0, \quad s x_{1}>a, \quad s \geq 2 .
$$

We only have to show that these inequalities are strict. Indeed, $F<0$ and (16) imply $h>0$ and $s x_{1}>a$, then (6) gives $s \geq 2$. In the case of $a=x_{1}$, (11) gives $x_{2}=$ $\cdots=x_{2 s+1}=0$, so $F=2 a h-h^{2} \geq 0$. For $x_{1}=0$, inequality (6) again gives $x_{2}=\cdots=x_{2 s+1}=0$ and $F=2 a h-h^{2}$. Finally, $a=h$ contradicts (14).

Define $k$ as the largest integer with $x_{1}=x_{2}=\cdots=x_{k}$. Then (11) and $s x_{1}>a$ from (17) give $k<s$. We claim that

$$
a-k x_{1}+k h>0 \text {. }
$$

Using the monotonicity and that $k<s$, then (11), and finally the positivity of $h$, one gets the equivalent form

$$
k x_{1}=x_{1}+\cdots+x_{k} \leq x_{1}+\cdots+x_{s} \leq a<a+k h .
$$

Now let $\eta>0$ be sufficiently small and define

$$
\begin{aligned}
& a^{\prime}=a-k \eta, \\
& x_{i}^{\prime}= \begin{cases}x_{i}-\eta & \text { for } \quad 1 \leq i \leq k, \\
x_{i} & \text { for } \quad k<i \leq 2 s+1,\end{cases} \\
& h^{\prime}=h \text { unchanged. }
\end{aligned}
$$

One can see that $k<2 s-1$ and (17) imply that the inequalities in Lemma 5 (i.e., (6), (10), (12), (13), and (15)) still hold for $\left(a^{\prime}, x_{1}^{\prime}, \ldots, x_{2 s+1}^{\prime}, h^{\prime}\right)$, and it is a feasible solution. 
However,

$$
\begin{aligned}
F\left(a, x_{1}, \ldots, x_{2 s+1}, h\right)-F\left(a^{\prime}, x_{1}^{\prime}, \ldots, x_{2 s+1}^{\prime}, h^{\prime}\right) \\
=\eta k\left(h-x_{2 s+1}\right)+\eta\left(a-k x_{1}+k h-2 k \eta\right)>0 .
\end{aligned}
$$

Here the first term is nonnegative by (13), and the second term is strictly positive by (18) if $\eta$ is sufficiently small and positive. This final contradiction completes the proof of Lemma 5 (and thus the proof of Lemma 4, too).

\section{Area Estimates}

Here we finish the proof of Theorem 2. We may suppose that the vertices of $\Delta_{0}$ are $(0,0)$, $(0,1)$, and $(1,0)$ and $\mathcal{H}$ consists of isosceles right triangles of sizes $x_{1} \geq \cdots \geq x_{n}>0$. Suppose that the integer $N$ is large enough, namely

$$
x_{n}>x_{1} / N
$$

and replace $\Delta_{1}$ by $N^{2}$ small triangles of sizes $x_{1} / N$. The system of $n-1+N^{2}$ triangles obtained is denoted by $\mathcal{H}^{N}$. First, we define an $\varepsilon$-covering of $\Delta_{0}$ by $\mathcal{H}^{N}$, and then we return to the case of $\mathcal{H}$ itself.

We form disjoint subgroups $\mathcal{H}_{1}, \mathcal{H}_{2}, \ldots, \mathcal{H}_{m}$ of $\mathcal{H}^{N}$, and at the same time we define right-angled trapezoids $T_{1}, T_{2}, \ldots, T_{m}$ such that some $\varepsilon$-translations of the members of $\mathcal{H}_{k}$ cover $T_{k}$ for $k=1,2, \ldots, m$. ( $T_{m}$ is a degenerate trapezoid, i.e., a triangle.) These trapezoids are obtained by cutting $\Delta_{0}$ by horizontal lines, they are packed on each other, and $T_{1}$ lies on the bottom (i.e., adjacent to the $x$-axis). The base lengths of $T_{k}$ are denoted by $a_{k}$ and $a_{k+1}, a_{1}=1>a_{2}>\ldots>a_{m}>a_{m+1}=0$, its height is $h_{k}, h_{k}=a_{k}-a_{k+1}$ and side lengths are $h_{k}$ and $\sqrt{2} h_{k}$, with vertices $\left(0,1-a_{k}\right),\left(0,1-a_{k+1}\right),\left(a_{k}, 1-a_{k}\right)$, and $\left(a_{k+1}, 1-a_{k+1}\right)$. Finally, the groups $\mathcal{H}_{k}$ consist of smaller and smaller triangles, i.e.,

$$
\min _{\Delta \in \mathcal{H}_{k}} x(\Delta) \geq \max _{\Delta \in \mathcal{H}_{k+1}} x(\Delta)
$$

where $x(\Delta)$ stands for the side length of $\Delta$. However, the heights of trapezoids do not necessarily form a monotone sequence. We also maintain

$$
\sum_{\Delta \in \mathcal{H}^{N} \backslash\left(\mathcal{H}_{1} \cup \ldots \cup \mathcal{H}_{k-1}\right)} 2 \operatorname{Area}(\Delta) \geq a_{k}^{2}+a_{k} \max _{\Delta \in \mathcal{H}^{N} \backslash\left(\mathcal{H}_{1} \cup \ldots \cup \mathcal{H}_{k-1}\right)} x(\Delta) .
$$

This condition is very similar to (4); $x_{1}$ stands for $x_{2}$.

Our starting case is $k=1$. Then $\max x\left(\Delta_{i}\right)=x_{2}$ and $\sum x_{i}^{2}=\sum_{\Delta \in \mathcal{H}} 2 \operatorname{Area}(\Delta) \geq$ $a_{1}^{2}+a_{1} x_{2}$. This last inequality implies that $\sum x_{i} \geq\left(a_{1}^{2}+a_{1} x_{2}\right) / x_{2} \geq 2 a_{1}$ so conditions (6) and (7) hold, and Lemma 4 can be applied to $\mathcal{H}^{N}$ and $\Delta_{0}$. Then either the lemma supplies an $\varepsilon$-cover of $\Delta_{0}$ (in the case of $h \geq a_{1}$ ), or one obtains a set of triangles $\mathcal{H}_{1} \subset \mathcal{H}^{N}$ and a trapezoid $T_{1}$ with horizontal sides $a_{1}(=1)$ and $a_{2}$, and height $h_{1}:=h$ such that $\mathcal{H}_{1} \varepsilon$-cover $T_{1}$ and the induction hypotheses (19) and (20),

$$
\sum_{i>2 s+1} 2 \operatorname{Area}\left(\Delta_{i}\right) \geq\left(a_{1}-h\right)^{2}+\left(a_{1}-h\right) x_{2 s+2}
$$


are preserved for the rest of $\Delta_{0}$ with the rest of $\mathcal{H}^{N}$. In general, if $\mathcal{H}_{1}, \ldots, \mathcal{H}_{k-1}$ are already defined, then Lemma 4 either finishes the procedure by completing an $\varepsilon$-cover of $\Delta_{0}$, and then $m=k$, or supplies $\mathcal{H}_{k}$ and $T_{k}$ satisfying the induction hypotheses for $\mathcal{H}^{N} \backslash\left(\mathcal{H}_{1} \cup \cdots \cup \mathcal{H}_{k}\right)$ and $\Delta_{0} \backslash\left(T_{1} \cup \cdots \cup T_{k}\right)$. Since $\mathcal{H}^{N}$ has finitely many members, our induction procedure results in a full $\varepsilon$-translation covering of $\Delta_{0}$.

Since the smallest $N^{2}$ triangles are used at last (if they are used at all, in the above $\varepsilon$-cover), they are contained in the subfamilies $\mathcal{H}_{\ell}, \mathcal{H}_{\ell+1}, \ldots, \mathcal{H}_{m}$. Among these, $\mathcal{H}_{\ell+1}, \ldots, \mathcal{H}_{m}$ consist purely of triangles of sizes $x_{1} / N$. Hence $T_{\ell+1} \cup \cdots \cup T_{m}$ is contained in a triangle $\Delta_{1}^{\prime}$ of size $x_{1}$, defined by the vertices $(0,1),\left(0,1-x_{1}\right)$, and $\left(x_{1}, 1-x_{1}\right)$. It is easy to see that the $x_{1} / N$-sized members of $\mathcal{H}_{\ell}$, apart from one or two exceptions, are all in the $2 x_{1} / N$ neighborhood of $\Delta_{1}^{\prime}$.

Consider the coverings by $\mathcal{H}^{N}, \mathcal{H}^{N+1}$, etc. It is not difficult to see that the positions of $\Delta_{2}, \ldots, \Delta_{n}$ converge to a limit. The above considerations show that in that limit position they $\varepsilon$-cover $\Delta_{0} \backslash \Delta_{1}^{\prime}$. Place $\Delta_{1}$ onto $\Delta_{1}^{\prime}$ to obtain an $\varepsilon$-translation covering of $\Delta_{0}$ by $\mathcal{H}$.

\section{6. $\rho$-Covering of the Isosceles Right Triangle, Starting the Induction}

We prove Theorem 3 by induction on $n$. Suppose that $R_{i}=x_{i} R_{0}, x_{1} \geq \cdots \geq x_{n}>0$. In the case of $n=1$, (5) implies that $x_{1} \geq \sqrt{c}>1$ so it certainly can $\rho$-cover $R_{0}$. If $x_{2} \leq(c-1)=0.2071 \ldots$ then Theorem 2 gives an $\varepsilon$-cover and we are done. To avoid using many subscripts, we sometimes use $x, y$, and $z$ for $x_{1}, x_{2}$ and $x_{3}$, respectively. From now on, we suppose that $y:=x_{2} \geq(c-1)=0.2071 \ldots$, and we may also suppose that the size of $R_{0}$ is 1 , that is $a=1$.

In this section we deal with two cases, using induction if $x>0.547$ (Step 1), and if $0.6>x>0.378$ (Step 2). In the next section we eliminate the cases $0.38 \geq x>0.336$ (Step 3) and $x>0.234$ (Step 5). Here we used computers. Finally, in Section 8, with a different method we finish the induction by investigating the case $x_{1} \leq \frac{1}{4}$.

Step 1. $x \geq 0.547$

Put $R_{1}$ into $R_{0}$ such that a leg of $R_{1}$ lies on the hypotenuse of $R_{0}$ and splits it into two segments of lengths $x$ and $\sqrt{2}-x$ (see Fig. 3). The set $R_{0} \backslash R_{1}$ can be covered by an isosceles right triangle $R^{\prime}$ of size $\sqrt{2}-x$. Hence one can use the induction hypothesis
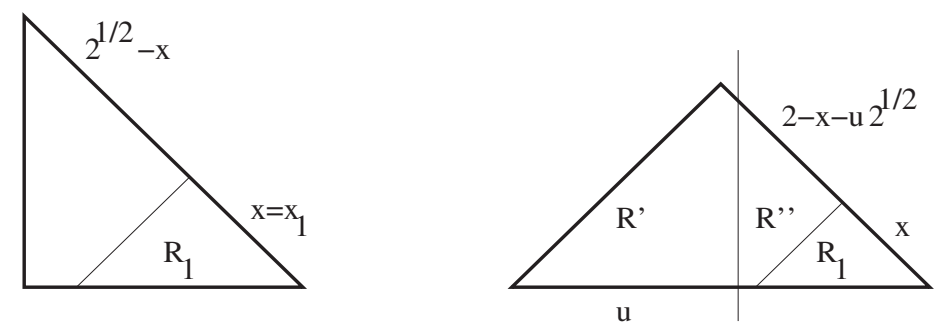

Fig. 3. The cases $x>0.547$ and $0.6>x>0.378$. 
for $R^{\prime}$ and for the remaining $(n-1)$ triangles $\left\{R_{2}, \ldots, R_{n}\right\}$ if

$$
\sum_{i \geq 2} x_{i}^{2} \geq c(\sqrt{2}-x)^{2} \text {. }
$$

Here the left-hand side is at least $c-x^{2}$, so (21) holds if $c-x^{2} \geq c(\sqrt{2}-x)^{2}$. Thus it holds for $1 \geq x \geq(1+\sqrt{8}) / 7=0.5469 \ldots$.

Step 2. $0.6>x \geq 0.378$

Put $R_{1}$ into $R_{0}$ in homothetic position sharing a vertex other than the apex (see again Fig. 3). Then $R_{0} \backslash R_{1}$ can be covered by two triangles $R^{\prime}$ and $R^{\prime \prime}$ of sizes $u$ and $2-x-u \sqrt{2}$, respectively, for any choice of $u$.

Select a subset of triangles $\mathcal{H}^{\prime} \subset\left\{R_{2}, \ldots, R_{n}\right\}$ and let $\mathcal{H}^{\prime \prime}$ be the rest of the triangles. Define $u$ such that $\sum_{R \in \mathcal{H}^{\prime}} 2 \operatorname{Area}(R)=: c u^{2}$. Then the induction hypothesis implies that $\mathcal{H}^{\prime}$ can $\rho$-cover $R^{\prime}$. If the rest of the triangles have sufficiently large total areas (at least $c$ Area $\left(R^{\prime \prime}\right)$ ), then induction can be applied to $\mathcal{H}^{\prime \prime}$ and $R^{\prime \prime}$ and we are done. A sufficient condition for this is that

$$
c-x^{2}-c u^{2} \geq c(2-x-u \sqrt{2})^{2} .
$$

This holds for $u_{1} \leq u \leq u_{2}$, where

$$
u_{1,2}=\frac{1}{3}\left(\sqrt{2}(2-x) \mp \sqrt{-(2-x)^{2}+3\left(1-x^{2} / c\right)}\right) .
$$

Suppose now that $u_{1}$ and $u_{2}$ exists. We would like to select $\mathcal{H}^{\prime}$ such that $u$ gets into the above range, i.e.,

$$
c u_{1}^{2} \leq \sum_{R_{i} \in \mathcal{H}^{\prime}} x_{i}^{2} \leq c u_{2}^{2} .
$$

Select the triangles into $\mathcal{H}^{\prime}$ one by one but in a fixed order, say, $x_{2}, x_{3}, \ldots$ If the difference of the left-hand side and the right-hand side of (23) is at least $x^{2}$, then the sequence

$$
0, x_{2}^{2}, x_{2}^{2}+x_{3}^{2}, x_{2}^{2}+x_{3}^{2}+x_{4}^{2}, \ldots, \sum_{2 \leq i \leq n} x_{i}^{2} \quad\left(\geq c-x^{2} \geq c u_{1}^{2}\right)
$$

cannot jump the gap $\left[c u_{1}^{2}, c u_{2}^{2}\right]$, consequently, a partition $\mathcal{H}^{\prime}, \mathcal{H}^{\prime \prime}$ satisfying (22) exists.

Thus, we can use induction if $c u_{2}^{2}-c u_{1}^{2} \geq x^{2}$. Rearranging, this is equivalent to

$$
c^{2} \frac{32}{81}(2-x)^{2}\left(-(2-x)^{2}+3\left(1-\frac{x^{2}}{c}\right)\right)-x^{4} \geq 0 .
$$

One can easily see (e.g., by using Maple V as the author did) that this fourth-degree polynomial is indeed positive for $0.378 \leq x<0.6$.

\section{Gluing $R_{1}, R_{2}$, and $R_{3}$}

In this section we continue the proof of Theorem 3. The method of Step 2 can be summarized in the following lemma. Note that (like in Fig. 3) $R_{0}$ can always be $\rho$ covered by three triangles $R^{\prime}, R^{\prime \prime}$, and $R^{\prime \prime \prime}$ of sizes $u, 2-v-u \sqrt{2}$, and $v$, respectively. 
Lemma 6. Let $\mathcal{H}^{\prime \prime \prime} \subset \mathcal{H}$ be a set of triangles with total area $A / 2$, and suppose that $\mathcal{H}^{\prime \prime \prime}$ can $\rho$-cover a triangle $R^{\prime \prime \prime}$ of size $v$. Let $\max \left\{x(R): R \in \mathcal{H} \backslash \mathcal{H}^{\prime \prime \prime}\right\} \leq w$. Suppose that

$$
c^{2} \frac{32}{81}(2-v)^{2}\left(-(2-v)^{2}+3\left(1-\frac{A}{c}\right)\right)-w^{4} \geq 0 .
$$

Then there exists a partition $\mathcal{H}^{\prime} \cup \mathcal{H}^{\prime \prime}$ of $\mathcal{H} \backslash \mathcal{H}^{\prime \prime \prime}$ and a real number $u$, such that $\sum_{R \in \mathcal{H}^{\prime}} 2 \operatorname{Area}(R)=c u^{2}$ and $\sum_{R \in \mathcal{H}^{\prime \prime}} 2 \operatorname{Area}(R) \geq c(2-v-u \sqrt{2})^{2}$.

Therefore, the induction hypothesis can be applied to $\mathcal{H}^{\prime}$ and $R^{\prime}$ and to $\mathcal{H}^{\prime \prime}$ and $R^{\prime \prime}$. These together with $\mathcal{H}^{\prime \prime \prime}$ yield a $\rho$-cover of $R_{0}$.

Step 3. $0.38>x \geq 0.336$

In Step 2 we used Lemma 6 for $\mathcal{H}^{\prime \prime \prime}=\left\{R_{1}\right\}, v=x$ and $A=x^{2}$. This time $\mathcal{H}^{\prime \prime \prime}=$ $\left\{R_{1}, R_{2}\right\}$, i.e., we glue $R_{1}$ and $R_{2}$ together so that they can cover an isosceles right triangle $R^{\prime \prime \prime}$ of size $v:=(x+y) / \sqrt{2}$. Then put $R^{\prime \prime \prime}$ into $R_{0}$ in homothetic position sharing a vertex other than the apex. We can use induction applying Lemma 6 with $A:=x^{2}+y^{2}$ and $w:=y^{2}$. Substituting to the condition (24) we get

$$
c^{2} \frac{32}{81}\left(2-\frac{x+y}{\sqrt{2}}\right)^{2}\left(-\left(2-\frac{x+y}{\sqrt{2}}\right)^{2}+3\left(1-\frac{x^{2}+y^{2}}{c}\right)\right)-y^{4} \geq 0 .
$$

One can easily see (e.g., by using Maple V), that this fourth-degree polynomial is indeed positive for $(x, y) \in D$, where $D$ is a quadrilateral defined by the vertices $(0.262,0.262),(0.5,0.5),(0.5, c-1)$, and $(0.336, c-1)$. Especially, from now on, we may suppose that

$$
(c-1) \leq y \leq x \leq 0.336, \quad y \leq 0.262
$$

Step 4. The case of small $z$

Replace $R_{2}$ by another copy of $R_{3}$. Then the system obtained $\varepsilon$-covers $R_{0}$ if Theorem 2 can be applied. This Theorem applies if the new system still has a total area at least $(1+z)$ Area $\left(R_{0}\right)$, i.e., if $1+z \leq c-y^{2}+z^{2}$. So from now on, we may suppose that the opposite holds:

$$
(1-c)+z-z^{2}+y^{2} \geq 0 .
$$

Step 5. $0.234<x \leq 0.336$

Let $C \subset \mathbf{R}^{3}$ be the set of those (x,y,z) points in 3-space which satisfy (25), (26) and $0.234 \leq x$. In this section we apply induction if $\left(x_{1}, x_{2}, x_{3}\right) \in C$.

Since $y \leq 0.262$ we get from (26) that $z>0.167$, so the sizes of $R_{1}, R_{2}$ and $R_{3}$ are relatively close to each other. As before, attach the apex vertices of $R_{2}$ and $R_{3}$ so that a leg of $R_{3}$ is part of a leg of $R_{2}$. Then they can cover an isosceles right triangle $R^{*}$ of size $(y+z) / \sqrt{2}$. Then again glue $R^{*}$ and $R_{1}$ together so that they can cover an isosceles right triangle $R^{\prime \prime \prime}$ of size $x / \sqrt{2}+(y+z) / 2$ (see Fig. 4). 

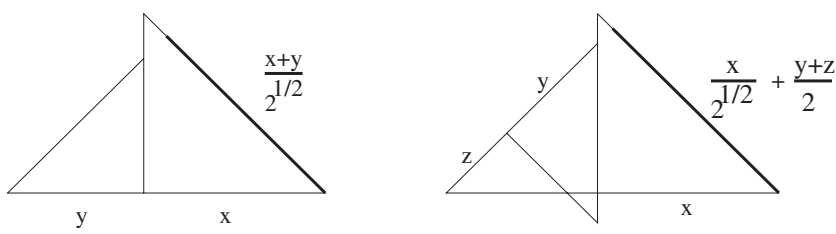

Fig. 4. Gluing $R_{1}$ and $R_{2}$, and gluing $R_{1}, R_{2}$, and $R_{3}$.

Apply Lemma 6 with $\mathcal{H}^{\prime \prime \prime}:=\left\{R_{1}, R_{2}, R_{3}\right\}, v:=2-x / \sqrt{2}-(y+z) / 2, A:=$ $x^{2}+y^{2}+z^{2}$, and $w:=z^{4}$. Substitute these values to (24):

$c^{2} \frac{32}{81}\left(2-\frac{x}{\sqrt{2}}-\frac{y+z}{2}\right)^{2}\left(-\left(2-\frac{x}{\sqrt{2}}-\frac{y+z}{2}\right)^{2}+3\left(1-\frac{x^{2}+y^{2}+z^{2}}{c}\right)\right)-z^{4}$.

One can easily see (e.g., by using Maple V) that this fourth-degree polynomial is indeed positive for $(x, y, z) \in C$. The calculations can be reduced to two variables, because if we start in any feasible point $(x, y, z) \in C$ and move in direction $(1,-\sqrt{2}, 0)$ (or its opposite direction) then (27) decreases. Hence the minimum is taken on the boundary of $C$.

Especially, from now on, we may suppose that $x \leq 0.234$.

\section{8. $\rho$-Covering a Strip, the End of the Induction}

In this section we finish the induction proof of Theorem 3. We need a lemma.

Lemma 7. Suppose that $P$ is a (finite) set of positive reals, $c, q>0$, such that

$$
\sum_{p \in P} p^{2} \geq c, \quad \max P=p_{1}, \quad 2 p_{1} \leq c(1-q) .
$$

Then there exists a subset $S \subset P$ with

$$
\sum_{p \in S} p \geq 2 \quad \text { and } \quad \frac{\min _{p \in S} p}{\max _{p \in S} p} \geq q .
$$

Proof. Here $p_{1}, c$, and $q$ can be any reals, but we will use it for $p_{1}=0.234, c=$ $(\sqrt{2}+1) / 2=1.207 \ldots$, and $q=0.61$.

Consider the infinite region $C$ on the positive quadrant of the plane defined as the union of rectangles of dimensions $2 \times p_{1} q^{i}, i=0,1,2, \ldots$, and placed on the segment $(2 i, 0),(2 i+2,0)$. The area of $C$ is $2 p_{1} /(1-q)$.

Next, order the members of $P, p_{1} \geq p_{2} \geq \cdots$ and place squares of these sizes next to each other on the $x$-axis in the positive quadrant, i.e., let $D$ be the union of squares with base segments defined by the vertices $\left(p_{1}+\cdots+p_{i-1}, 0\right)$ and $\left(p_{1}+\cdots+p_{i-1}+p_{i}, 0\right)$.

Since $\operatorname{Area}(C) \leq \operatorname{Area}(D)$, there is a segment $(2 i, 0),(2 i+2,0)$ which meets a square of size exceeding $p_{1} q^{i}$. Suppose $i$ is the smallest with this property. Here $i \geq 1$. 
Then $S$ can consists of the sizes of those squares in $D$ having a common point with the segment $(2 i-2,0),(2 i, 0)$.

Step 6. $x \leq 0.234$

Now we return to the proof of Theorem 3 . Apply Lemma 7 for the set $P=\left\{x_{1}, x_{2}, \ldots, x_{n}\right\}$ with $q=0.61$. We obtain a subset $S=\left\{y_{1}, y_{2}, \ldots, y_{m}\right\} \subset P$ such that

$$
0.234>y:=y_{1} \geq y_{2} \geq \cdots \geq y_{m}>0.61 y
$$

and

$$
y_{1}+\cdots+y_{m} \geq 2 .
$$

The rest of the procedure is similar to the one in Section 3. Our aim is, like in Lemma 4, to define a trapezoid $T$ with vertices $(0,0),(0, h),(1,0)$, and $(1-h, h)$ and to find an $s \geq 1$ such that

(P1) the triangles of sizes $y_{1}, \ldots, y_{2 s+1}$ can $\varepsilon$-cover $T$ (here $2 s+1 \leq m$ ), and

(P2) $c \operatorname{Area}(T) \geq \frac{1}{2} \sum_{1 \leq i \leq 2 s+1} y_{i}^{2}$.

Then one can apply induction, the rest of the triangles can $\rho$-cover $R_{0} \backslash T$.

Let $g:=0.61 y$. Consider the triangles of sizes $y_{2 i}$ and $y_{2 i+1}$, turn them so that they touch each other at their hypotenuse and together they cover a rectangle $Q_{i}$ of height $g$ and base $y_{2 i}+y_{2 i+1}-g$. Note that the base of $Q_{i}$ is at least $y_{2 i} \geq g$ and at most $2 y-g=1.39 y$.

Define $s$ as the smallest integer satisfying

$$
1 \leq y_{1}+\sum_{1 \leq i \leq s}\left(y_{2 i}+y_{2 i+1}-g\right) .
$$

We claim that such an $s$ exists, $7 \leq 2 s+1 \leq m$. Indeed, we get $1 \leq(1+1.39 s) y \leq$ $(1+1.39 s) 0.234$, this implies $s \geq 3$. On the other hand, by definition

$$
1>y_{1}+\sum_{1 \leq i \leq s-1}\left(y_{2 i}+y_{2 i+1}-g\right) .
$$

This implies that

$$
1-y_{1}>y_{2}+y_{4}+\cdots+y_{2 s-2} \geq y_{3}+y_{5}+\cdots+y_{2 s-1},
$$

so $2-y_{1}>\sum_{i \leq 2 s-1} y_{i}$. Thus at least two more $y_{i}$ 's are needed to increase this sum to at least 2. Then (29) guarantees that these two positive $y_{i}$ 's exist, and thus the sum in (30) will reach 1 after finitely many steps.

Similarly as in Section 3, we define the height $h$ of the trapezoid $T$ by the equation

$$
1=y_{1}+\sum_{1 \leq i \leq s}\left(y_{2 i}+y_{2 i+1}-h\right)
$$

Then the triangles of sizes $y_{1}, \ldots, y_{2 s+1} \varepsilon$-cover $T$. Comparing (30) and (32) we obtain that

$$
y_{2 s+1} \leq h
$$


We have to show that these inequalities imply (P2), i.e.,

$$
\operatorname{ch}(2-h) \geq \sum_{1 \leq i \leq 2 s+1} y_{i}^{2} .
$$

Divide by $c$, add $((c-1) / c) \sum y_{i}^{2}$, rearrange, and apply (32). We get that the above inequality is equivalent to

$$
\begin{aligned}
L:=\frac{c-1}{c} \sum_{1 \leq i \leq 2 s+1} y_{i}^{2} & \geq-h(2-h)+\sum y_{i}^{2} \\
& =-h\left(2 \sum y_{i}-2 s h-h\right)+\sum y_{i}^{2}=\sum_{1 \leq i \leq 2 s+1}\left(y_{i}-h\right)^{2} .
\end{aligned}
$$

Define the function $p(t)$ as $\sum_{1 \leq i \leq 2 s+1}\left(y_{i}-t\right)^{2}$. Then

$$
L \geq p(t) \quad \text { for } \quad(2-\sqrt{2}) y_{1} \leq t \leq \sqrt{2} y_{2 s+1} .
$$

Indeed, $L \geq p(t)$ if $((c-1) / c) y_{i}^{2} \geq\left(y_{i}-t\right)^{2}$ for each $i$. Since $(c-1) / c=(\sqrt{2}-1)^{2}$, this holds for $(\sqrt{2}-1) y_{i} \geq\left|t-y_{i}\right|$, i.e., if $(2-\sqrt{2}) y_{i} \leq t \leq \sqrt{2} y_{i}$.

We have the lower bound $(2-\sqrt{2}) y_{1}<g \leq h$ by (33). Then (35) implies that $L \geq p(h)$ certainly holds for

$$
h \leq y_{2 s+1} \sqrt{2}
$$

Subtract (31) from (32), and again use the monotonicity. We obtain

$$
0<y_{2 s}+y_{2 s+1}-s h+(s-1) g \leq y-s h+s y_{2 s+1},
$$

yielding $h<y_{2 s+1}+(y / s)$. Since $y \leq y_{2 s+1} / 0.61$ we get $h \leq \sqrt{2} y_{2 s+1}$ for $s \geq 4$. Therefore (36) holds for $s \geq 4$ and we are done.

The last remaining case is $s=3$. We have $L \geq p(g)$ by (35), so our proof is complete if we show that $p(g) \geq p(h)$.

It is easy to see that the second-degree polynomial $p(t)$ takes its minimum at $\bar{y}:=$ $\left(\sum_{i} y_{i}\right) /(2 s+1)$. We have $g \leq \bar{y}$, so $p(t) \leq p(g)$ for all $t \in[g, 2 \bar{y}-g]$. So we are done if $h \leq 2 \bar{y}-g$. Using (32) this is equivalent to

$$
h+(2 s+1) g \leq 2 .
$$

Our last task is to prove that this always holds for the case $s=3, y<\frac{1}{4}$. Then (32) gives $h \leq(7 y-1)<\frac{1}{4}$, so $h+7 g<2$ follows from $g<y<\frac{1}{4}$.

\section{Conclusion, Remarks}

Note that our theorem holds for infinite sets of triangles satisfying (5).

Moon and Moser's result was extended by Groemer [8] and Bezdek and Bezdek [3] to higher dimensions proving that $f\left(Q^{(d)}\right)=2^{d}-1$, where now $Q^{(d)}$ is the $d$-dimensional cube. 
An algorithm for packing or covering a given set $K$ with a sequence of sets $\left\{C_{i}\right\}$ is an on-line method if the sets $C_{i}$ are given in sequence, and $C_{i+1}$ is presented only after $C_{i}$ has been put in place, without the option of changing the placement afterward. Januszewski et al. [11] proved that in Euclidean $d$-space, every sequence of cubes of total volume greater than or equal to $2^{d}+3$ can cover the unit cube in the on-line manner. This volume bound is astoundingly good, considering the best possible bound of $2^{d}-1$ for the analogous off-line problem.

A recent study on square coverings is by Abbott and Kathchalski [1].

L. Fejes Tóth conjectured that $2 \leq f(C) \leq 3$ for every planar disk, and $f(D)=\frac{9}{4}$ for the circular disks (and for ellipses).

Concerning our computer-aided proof of Theorem 3, with a little work Step 6 can be extended to cover all cases $x_{1} \leq 0.25$, but this does not simplify the previous steps.

In [16] it was claimed that $f_{\rho}(R) \geq c=(1+\sqrt{2}) / 2$ where $R$ is the right isosceles triangle, but their argument seems to be rather incomplete. We conjecture more, that any placement with any rotations of the triangles of sizes

$$
(1-\eta)(\sqrt{2}-1)^{k}, \quad \text { where } k=0,1,2, \ldots,
$$

cannot cover $R$.

There are many covering results where only a few number of smaller pieces can be used, see, e.g., some recent works of M. Lassak et al. These are obviously related to Hadwiger's conjecture and Borsuk's problem, see the recent problem book by Brass et al. [4].

\section{Acknowledgments}

The author is greatly thankful to I. Bárány and M. Simonovits for helpful conversations.

\section{References}

1. H. L. Abbott and M. Katchalski: Covering squares with squares. Discrete Comput. Geom. 24 (2000), 151-169.

2. A. S. Besicovitch: Measure of asymmetry of convex curves. J. London Math. Soc. 23 (1948), 237-240.

3. A. Bezdek and K. Bezdek: Eine hinreichende Bedingung für die Überdeckung des Einheitswürfels durch homothetische Exemplare im $n$-dimensionalen euklidischen Raum. (German) [A sufficient condition for the covering of the unit cube by homothetic copies in the $n$-dimensional Euclidean space.] Beiträge Algebra Geom. 17 (1984), 5-21.

4. P. Brass, W. Moser, and J. Pach: Research Problems in Discrete Geometry. Springer-Verlag, New York, 2005.

5. I. Fáry: Sur la densité des réseaux de domaines convexes. (French) Bull. Soc. Math. France 78 (1950), 152-161.

6. L. Fejes Tóth: Lagerungen in der Ebene auf der Kugel und im Raum. (German) Springer-Verlag, Berlin, 1972.

7. Z. Füredi: Covering a triangle with homothetic copies. Discrete Geometry, pp. 435-445. Monogr. Textbooks Pure Appl. Math., 253, Dekker, New York, 2003.

8. H. Groemer: Covering and packing properties of bounded sequences of convex sets. Mathematika 29 (1982), 18-31. 
9. J. Januszewski: Covering a triangle with sequences of its homothetic copies. Period. Math. Hungar. 36 (1998), 183-189.

10. J. Januszewski: Translative covering a convex body by its homothetic copies. Studia Sci. Math. Hungar. 40 (2003), 341-348.

11. J. Januszewski, M. Lassak, G. Rote, and G. Woeginger: On-line $q$-adic covering by the method of the $n$th segment and its application to on-line covering by cubes. Beiträge Algebra Geom. 37 (1996), 51-65.

12. J. W. Moon and L. Moser: Some packing and covering theorems. Colloq. Math. 17 (1967), 103-110.

13. J. Pach and P. K. Agarwal: Combinatorial Geometry, Wiley, New York, 1995.

14. É. Vásárhelyi: Über eine Überdeckung mit homothetischen Dreiecken. (German) [On a covering with homothetic triangles.] Beiträge Algebra Geom. 17 (1984), 61-70.

15. É. Vásárhelyi: Covering of a triangle by homothetic triangles. Studia Sci. Math. Hungar. 28 (1993), 163-172.

16. C. Xu, L. Yuan, and R. Ding: Covering isosceles right triangles with isosceles right triangles. Geombinatorics 14 (2005), 194-199.

Received October 17, 2006, and in revised form October 24, 2006. Online publication July 17, 2007. 